confined in several occupations. Thus, we aimed to evaluate PAHs exposure across a wide range of occupations using its urinary metabolite 1-hydroxypyrene (1OHP).

Methods To evaluate PAHs exposure across occupations, we collected the urine 1-OHP data from the Korean National Environmental Health Survey which is a nationwide biomonitoring survey. The data contained information about urine 1-OHP levels, cigarette smoking status, and standard occupational codes. We calculated summary statistics of urine 1-OHP levels for each occupation. In addition, we calculated the relative exposure indicators which are the proportions of exceeding the quartile levels. Since cigarette smoking is a single most influential factor of PAHs exposure, we repeated the analyses by excluding current smokers.

Results Overall geometric means (GM) of all populations and non-smoker populations were $0.13 \mu \mathrm{g} / \mathrm{L}$ and $0.10 \mu \mathrm{g} / \mathrm{L}$, respectively. For the major group of occupation, 'Craft and Related Trades Workers' and 'Equipment, Machine Operating and Assembling Workers' showed the highest urine 1OHP levels, while 'Homemaker' showed the lowest level. For the sub-major group of occupation, 'Video and Telecommunications Equipment Related Occupations' showed the highest percentage (61\%) of exceeding the third quartile (Q3) level of all populations. While 'Legal and Administration Professional Occupations' showed the lowest percentage of exceeding the Q3 level of all populations. For the minor group of occupation, 'Horticultural and Landscape Workers' showed the highest percentage (64\%) of exceeding the Q3 level of all populations. While 'Kindergarten teachers' showed the lowest percentage of exceeding the Q3 level of all populations.

Conclusions Our results will provide ancillary information about PAHs exposure across occupations, especially in occupations where PAHs exposure has not well known.

\section{O6D.4 ASSOCIATION OF OCCUPATIONAL EXPOSURES WITH EX VIVO FUNCTIONAL IMMUNE RESPONSE IN WORKERS HANDLING CARBON NANOTUBES AND NANOFIBERS}

\begin{abstract}
${ }^{1,2}$ Mary Schubauer-Berigan*, ${ }^{2}$ Matthew Dahm, ${ }^{3}$ John Beard, ${ }^{4}$ Vamsi Kodali, ${ }^{4}$ Patti ZeidlerErdely, ${ }^{4}$ Aaron Erdely. ${ }^{1}$ International Agency for Research on Cancer, Lyon, France; ${ }^{2}$ National Institute for Occupational Safety and Health, Cincinnati, USA; ${ }^{3}$ Brigham Young University, Provo, USA; ${ }^{4}$ National Institute for Occupational Safety and Health, Morgantown, USA
\end{abstract}

\subsection{6/OEM-2019-EPI.156}

Animal toxicology studies suggest that workers exposed to carbon nanotubes or nanofibers (CNT/F) may experience pulmonary or systemic health effects; however, direct human evidence is lacking. Our study's objective was to evaluate associations between $\mathrm{CNT} / \mathrm{F}$ exposure and ex vivo responses of leukocytes challenged with secondary stimulants, adjusting for potential confounders, in a cross-sectional study. We measured multi-day exposure using $\mathrm{CNT} / \mathrm{F}$ structure count (SC) and elemental carbon air concentrations among 102 U.S. workers. Demographic, lifestyle, and other occupational information was obtained via in-person interview. Workers' whole blood was incubated for 18 hours with and without two microbial stimulants (lipopolysaccharide and staphylococcal enterotoxin type B) using TruCulture ${ }^{\circledR}$ technology to evaluate immune cell activity. Following incubation, collected supernatants were preserved and subsequently analyzed for cytokine and chemokine concentrations. The ratio of stimulant:null response for each protein was analyzed using multiple linear regression, principal components (PC) analysis, and Ingenuity ${ }^{\circledR}$ Pathway Analysis (IPA) to determine whether patterns of protein response were associated with $\mathrm{CNT} / \mathrm{F}$ exposure. We found that $\mathrm{CNT} / \mathrm{F}$ metrics (most consistently, the SCbased) were significantly $(\mathrm{p}<0.05)$ inversely associated with stimulant:null ratios of GM-CSF, IFN- $\gamma$, interleukin (IL)-2, IL-4, IL-5, IL-10, IL-17, and IL-23. CNT/F metrics were significantly inversely associated with PC1 (a weighted mean of most biomarkers that explained $25 \%$ of the variance in the set of protein ratios) and PC2 (a biomarker contrast that explained 14\%). Among other occupational exposures, only solvent exposure was significantly (and was inversely) related to PC2. IPA suggested a $\mathrm{CNT} / \mathrm{F}$-associated generalized inhibition of all leukocyte responses when challenged with a secondary stimulus. We found that $\mathrm{CNT} / \mathrm{F}$ exposure metrics were uniquely related to a pattern of reduced stimulant responses in challenged circulating leukocytes. This approach, if replicated in other exposed populations, may present a relatively sensitive method to evaluate human response to $\mathrm{CNT} / \mathrm{F}$ or other occupational exposures.

\section{D.5 ELECTRONIC WASTE RECYCLING EXPOSURE AND HORMONE LEVELS IN WORKERS} 1,2 Sabrina Gravel, ${ }^{2}$ Bouchra Bakhiyi, ${ }^{2,3}$ Jérôme Lavoué, ${ }^{2}$ Marc-André Verner, ${ }^{2}$ Joseph Zayed,
1,2 ${ }^{2}$ rance Labrèche*. ${ }^{1}$ Institut de recherche Robert-Sauvé en santé et en sécurité du travail
(IRSST), Montreal, Canada; ${ }^{2}$ Dept. Environmental and Occupational Health, School of Public
Health, University of Montreal, Montreal, Canada; ${ }^{3}$ Centre de recherche du Centre
hospitalier de I'Université de Montréal (CRCHUM), Montreal, Canada

\subsection{6/OEM-2019-EPI.157}

Background and objective Electronic waste recycling (e-recycling) exposes workers to several contaminants, including flame retardants that are suspected endocrine disruptors. We aimed to explore the association between polybrominated diphenyl ethers (PBDEs) and hormone levels in the serum of Canadian e-recycling workers.

Methods In a cross-sectional study, blood samples were collected from 85 e-recycling workers (six facilities) and from 15 workers in other types of recycling (two facilities), at the end of a work shift. Socio-demographic information was obtained by questionnaire and body mass index (BMI) was calculated from measured height and weight. Serum concentrations of 13 PBDE congeners were measured as well as thyroid hormones (free and total thyroxine [T4], triiodothyronine [T3], thyroid stimulating hormone [TSH]) and testosterone (free and total). Linear regressions were stratified on sex and adjusted for age, BMI, seniority, smoking status, and type of recycling. Ten participants were excluded because of thyroid or testicular problems.

Results Participants were 23 women and 77 men (mean 40 years old, $\mathrm{SD}=12$ years). Average hormone levels were within the laboratory normal range. In e-recycling, geometric mean concentrations of the most detected congeners were 11, 11 and $20 \mathrm{ng} / \mathrm{g}$ lipids for BDE47, BDE153 and BDE209, respectively. Only BDE209 concentrations were higher in e-recycling than in the control group. A two-fold increase in serum 